\section{Area Spectral Efficiency of Soft-Decision Space-Time-Frequency Shift-Keying-Aided Slow-Frequency-Hopping Multiple Access}

\author{
Hoang Anh Ngo, Student Member, IEEE, and \\ Lajos Hanzo, Fellow, IEEE
}

\begin{abstract}
Slow-frequency-hopping multiple access (SFHMA) can provide inherent frequency diversity and beneficially randomize the effects of cochannel interference. It may also be advantageously combined with our novel space-time-frequency shift keying (STFSK) scheme. The proposed system's area spectral efficiency is investigated in various cellular frequency reuse structures. Furthermore, it is compared to both classic Gaussian minimum shift keying (GMSK)-aided SFHMA and GMSKassisted time-division/frequency-division multiple access (TD/FDMA). The more sophisticated third-generation wideband code-division multiple access (WCDMA) and the fourth-generation Long Term Evolution (LTE) systems were also included in our comparisons. We demonstrate that the area spectral efficiency of the STFSK-aided SFHMA system is higher than the GMSK-aided SFHMA and TD/FDMA systems, as well as WCDMA, but it is only $60 \%$ of the LTE system.
\end{abstract}

Index Terms-Fading channel, frequency reuse, slow-frequencyhopping multiple access (SFHMA), space-time-frequency shift keying (STFSK), time-division/frequency-division multiple access (TD/FDMA).

\section{INTRODUCTION}

Slow-frequency-hopping multiple access (SFHMA) [1] not only provides inherent frequency diversity but also advantageously randomizes the effects of cochannel interference. Furthermore, it can avoid the problem of prolonged fades, which are typically experienced by stationary or slow-moving mobile stations (MSs), because hopping to another independently faded frequency might curtail fading. The classic SFH900 mobile system [2], [3], which was based on "mixed" slow frequency hopping $(\mathrm{SFH})$ combined with time division, demonstrated the benefits of SFHMA.

Due to their potential to increase the attainable system capacity without requiring additional bandwidth, multiple-input-multipleoutput (MIMO) techniques have extensively been investigated. The family of MIMO systems typically employs spatial-division multiplexing (SDM), such as the Bell Labs Layered Space-Time (BLAST) schemes in [4] and [5] for increasing the transmission rate or space-time codes (STCs) [6], [7] for maximizing the attainable diversity order. More recently, spatial modulation [8] and space shift keying [9] have been proposed to avoid any interantenna interference and synchronization. As a further advance, space-time-frequency shift keying (STFSK) [10] was designed to achieve a beneficial diversity gain, which may be gleaned from the following three different domains: 1) the space domain; 2) the time domain; and 3) the frequency-domain.

All the aforementioned techniques play an important role in the overall system design, but they have typically been investigated independently. Therefore, in this paper, we develop the philosophy of

Manuscript received April 18, 2011; revised August 31, 2011 and December 19, 2011; accepted December 21, 2011. Date of publication January 9, 2012; date of current version March 21, 2012. This work was supported in part by the European Union through the Seventh Framework Program Concerto Project under Grant FP7/2007-2013, Research Councils U.K. (RCUK) through the U.K.-India Advanced Technology Centre, and the China-U.K. Science Bridge in 4G Wireless Communications. The review of this paper was coordinated by Prof. Y. Su.

The authors are with the School of Electronics and Computer Science, University of Southampton, SO17 1BJ Southampton, U.K. (e-mail: han08r@ ecs.soton.ac.uk; 1h@ecs.soton.ac.uk).

Digital Object Identifier 10.1109/TVT.2012.2183627
STFSK into a multiuser multicell SFHMA system to investigate the interplay of these techniques on the performance of the holistically optimized system. The novelty and rationale of our proposed scheme is summarized as follows.

1) We intrinsically amalgamate STFSK with SFHMA and then investigate the achievable area spectral efficiency (ASE) of a realistic multiuser multicell wireless environment under different cellular frequency reuse structures.

2) The study in [11] showed that the SFHMA system is superior compared to the time-division/frequency-division multiple access (TD/FDMA) benchmarker for a low-complexity 16-kbit/s subband speech codec combined with Reed-Solomon (RS) coding. By contrast, in this paper, we demonstrate that the SFHMA system becomes inferior to the TD/FDMA system when stateof-the-art components such as the advanced multirate (AMR) speech codec and convolutional coding are employed.

3) We demonstrate that, by intrinsically amalgamating lowcomplexity STFSK and SFHMA, we attained an improved ASE compared to TD/FDMA and WCDMA. However, this ASE remains lower than the more complex Long Term Evolution (LTE) system when the same convolutional channel code and system bandwidth are employed.

This paper is outlined as follows. Section II briefly describes the STFSK philosophy, whereas the SFHMA's frequency reuse structures are presented in Section III. In Section IV, we investigate the spectral efficiency of the STFSK-aided SFHMA. Finally, our concluding remarks are offered in Section V.

\section{STFSK}

In this section, we will briefly describe the STFSK concept, which was detailed in [10]. Consider an $M \times N$-element system where the transmitter and the receiver employ $M$ and $N$ antennas, respectively. The channel is assumed to impose frequency-selective Rayleigh fading. Generally, a transmission block-based system model may be described as

$$
\boldsymbol{Y}(i)=\sum_{j=0}^{J-1} \boldsymbol{H}(i-j, j) \boldsymbol{S}(i-j)+\boldsymbol{V}(i)
$$

where $i$ indicates the block index, and $j$ represents the tap index in the tap-delay-line channel model, which consists of $J$ taps. Naturally, flat fading is encountered for $J=1$. Furthermore, $\boldsymbol{Y} \in \mathcal{C}^{N \times T}$ represents the received signals of the $N$ antennas, and $S \in \mathcal{C}^{M \times T}$ denotes the signal that is transmitted from $M$ antennas in $T$ time slots. Furthermore, $\boldsymbol{H}(i, j) \in \mathcal{C}^{N \times M}$ characterizes the coefficients of the $i$ th symbol at the $j$ th channel tap, each obeying correlated frequencyselective Rayleigh fading. Finally, $\boldsymbol{V}$ denotes the complex-valued zero-mean Gaussian distribution of $\mathcal{C} \mathcal{N}\left(0, N_{0}\right)$, where $N_{0}$ is the noise variance. It is also assumed that the fading and noise coefficients remain constant during each time slot.

Fig. 1 illustrates the transmitter of the STFSK scheme, where the information bits are divided into three parallel bits streams. In the first bit stream, each group of $\log _{2}(q)$ bits is mapped to one out of $Q$ predefined dispersion matrices $\boldsymbol{A}_{q} \in \mathcal{C}^{M \times T}(q=1,2, \ldots, Q)$. The second stream is mapped to $s_{l}(i)$ symbols $(l=1,2, \ldots, L)$ by a conventional modulation scheme such as $L$-ary phase-shift keying ( $L$-PSK) or $L$-ary quadrature-amplitude modulation ( $L$-QAM), whereas the third stream is mapped to the frequency shift keying (FSK) symbol $r(i)$, which is represented by $r(i)=\cos \left(2 \pi f_{r} t+\varphi_{r}\right)$, 


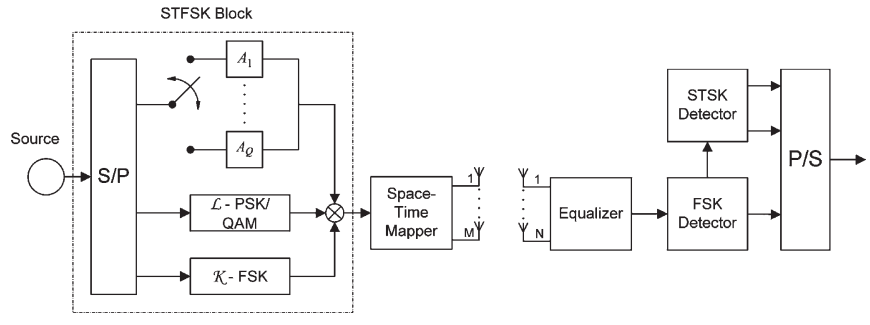

Fig. 1. Transceiver block diagram of the STFSK scheme.

where $\left.f_{i}(r=1,2, \ldots, K)\right)$ is the frequency that is associated with the $i$ th transmitted FSK symbol, and $\varphi_{i}$ is the random phase during the $i$ th symbol interval. Then, the resulting modulated streams are multiplexed to create the space-time-frequency block $\boldsymbol{S}(i) \in \mathcal{C}^{M \times T}$, which conveys a total of $\log _{2}(Q L K)$ source bits, yielding

$$
\boldsymbol{S}(i)=r(i) s(i) \boldsymbol{A}(i)
$$

where each symbol $s(i)$ is a function of time during the period $T_{s}$ of each time slot.

As shown in [10], the signal $\bar{Y}$ at the receiver may be presented in an element-wise form as

$$
\overline{\boldsymbol{Y}}(i)=\overline{\boldsymbol{H}}(i) \chi \boldsymbol{K}(i)+\overline{\boldsymbol{V}}(i)
$$

with the variables formulated as

$$
\begin{aligned}
\overline{\boldsymbol{Y}}(i) & =\operatorname{vec}(\boldsymbol{Y}(i)) \in \mathcal{C}^{N T \times 1} \\
\overline{\boldsymbol{H}}(i) & =\boldsymbol{I} \otimes \boldsymbol{H}(i) \in \mathcal{C}^{N T \times M T} \\
\overline{\boldsymbol{V}}(i) & =\operatorname{vec}(\boldsymbol{V}(i)) \in \mathcal{C}^{N T \times 1} \\
\chi & =\left[\operatorname{vec}\left(\boldsymbol{A}_{1}\right) \cdots \operatorname{vec}\left(\boldsymbol{A}_{Q}\right)\right] \in \mathcal{C}^{M T \times Q}
\end{aligned}
$$

where $\boldsymbol{I}$ is the $(T \times T)$-element identity matrix, and $\otimes$ is the Kronecker product. Furthermore, $\boldsymbol{K}(i) \in \mathcal{C}^{Q \times 1}$ is the equivalent transmitted signal vector, which may be expressed as

$$
\boldsymbol{K}(i)=[\underbrace{0, \ldots, 0}_{q-1}, r(i) s(i), \underbrace{0, \ldots, 0}_{Q-q}]^{T r}
$$

where $\operatorname{Tr}$ indicates the matrix transpose operation.

At the receiver, we employ an FSK demodulator that consists of a bank of $K$ parallel square-law detectors [12] to detect the activated frequencies of the SFSK symbols. Then, the maximum-likelihood (ML) detector [13] is employed to search for an appropriate pair of the $l$ th $(l=1, \ldots, L)$ PSK/QAM symbol and the $q$ th $(q=1, \ldots, Q)$ dispersion matrix. More particularly, the estimate $(\hat{q}, \hat{l}$,$) is given by$ minimizing the following metric:

$$
\begin{aligned}
(\hat{q}, \hat{l})=\arg \min _{q, l} \| & \overline{\boldsymbol{Y}}(i)-\overline{\boldsymbol{H}}(i) \chi \boldsymbol{K}_{q, l}(i) \\
& -\sum_{j=1}^{J-1} \overline{\boldsymbol{H}}(i-j, j) \chi \boldsymbol{K}(i-j) \|^{2} \\
=\arg \min _{q, l} \| \overline{\boldsymbol{Y}}(i)-s_{l}(i)(\overline{\boldsymbol{H}}(i) \chi)_{q} & \\
& -\sum_{j=1}^{J-1} \overline{\boldsymbol{H}}(i-j, j) \chi \boldsymbol{K}(i-j) \|^{2}
\end{aligned}
$$

where $s_{l}(i)$ is the $l$ th symbol in the $L$-point constellation at the $i$ th block index, and the signal vector $\boldsymbol{K}_{q, l}(1 \leq q \leq Q, 1 \leq l \leq L)$ is presented by

$$
\boldsymbol{K}_{q, l}(i)=[\underbrace{0, \ldots, 0}_{q-1}, r(i) s_{l}(i), \underbrace{0, \ldots, 0}_{Q-q}]^{T r} .
$$

Furthermore, $\sum_{j=1}^{J-1} \overline{\boldsymbol{H}}(i-j, j) \chi \boldsymbol{K}(i-j)$ represents the delayed paths of the dispersive channel, which is omitted in flat-fading environments, whereas $(\overline{\boldsymbol{H}}(i) \chi)_{q}$ denotes the $q$ th column of the matrix $\overline{\boldsymbol{H}}(i) \chi$.

Note that $M$ should be less than or equal to $T(M \leq T)$, because no further capacity gain may be achieved for $M>T$, as shown in [14] and [15]. In addition, to maintain a unity transmission power for a STSK symbol duration, each of the $Q$ dispersion matrices has to obey the power constraint of [16]

$$
\operatorname{tr}\left[\boldsymbol{A}_{q}^{\dagger} \boldsymbol{A}_{q}\right]=T \quad(q=1, \ldots, Q)
$$

where $\operatorname{tr}[\cdot]$ indicates the trace of a matrix, whereas the superscript $\dagger$ denotes the complex conjugate transpose operation.

\section{SFHMA}

The SFHMA technique employs SFH relying on unique userspecific pseudorandom hopping sequences to support multiple users. The SFH family may be divided into the following three categories: 1) orthogonal protocols; 2) random protocols; and 3) mixed protocols [11]. As a benefit of providing an increased freedom in system design and of its improved spectral efficiency [2], [3], the mixed protocol will be considered in the following sections.

The basic form of the reuse structure has three basic frequency-sets, also often referred to as colors [2], [3]. Each color is represented by one of the letters A, B, C, and each contains a set of $N_{c}$ frequencies. For a given reference cell that is associated with color $\mathrm{A}$, all other cells marked A are full-reuse cells, relying on the same set of frequencies. For the full-reuse structure, an active user in the reference cell will experience interference from an active user in the reuse cell during one of each sequence of $N_{c}$ hops when they happen to use the same frequency. Each additional active user from the same or other reuse cell will impose the same frequency collision probability, but at a different frequency in the reference user's sequence. Therefore, the so-called frequency collision rate is defined as the proportion of the hopping sequence when a 'hit' by an identical-frequency active user is encountered. For a full-reuse cluster size $C_{c}$, the frequency collision rate is equal to $C_{c} / 3 N_{c}$.

By contrast, in a fractional-reuse structure, each color is divided into $M_{c}$ overlapping subsets of $L_{c}$ groups of frequencies, which are referred to as 'shades' of that color or 'pseduocolor' [2]. Hence, the frequency-reuse cluster contains $3 M_{c}$ cells that are divided into $M_{c}$ subclusters of size 3 , each using a different shade of three colors. This approach is known as a $3 M_{c} / L_{c}$ fractional structure [2], where $L_{c} / M_{c}$ is the fraction of the $N_{c}$ frequencies that make up a color, which is constituted by the set of shades. Fig. 2 shows an example of a 21/3 reuse structure, where we have $M_{c}=7$ and $L_{c}=3$. Subsequently, the frequency collision rate is expressed as $y=\left(M_{c} k\right) /\left(L_{c} N_{c}\right)$, where $k$ is the fractional overlap factor. Note that we have $k=0$ for cells of different colors, whereas $k=1$ for cells of the same color and shade. Other values of $k$ may be found in [11].

\section{ASE OF STFSK-AIDED SFHMA}

\section{A. ASE of the SFHMA System}

In this section, the method proposed in [3] and [11] will be employed to evaluate the ASE of the proposed STFSK-aided 


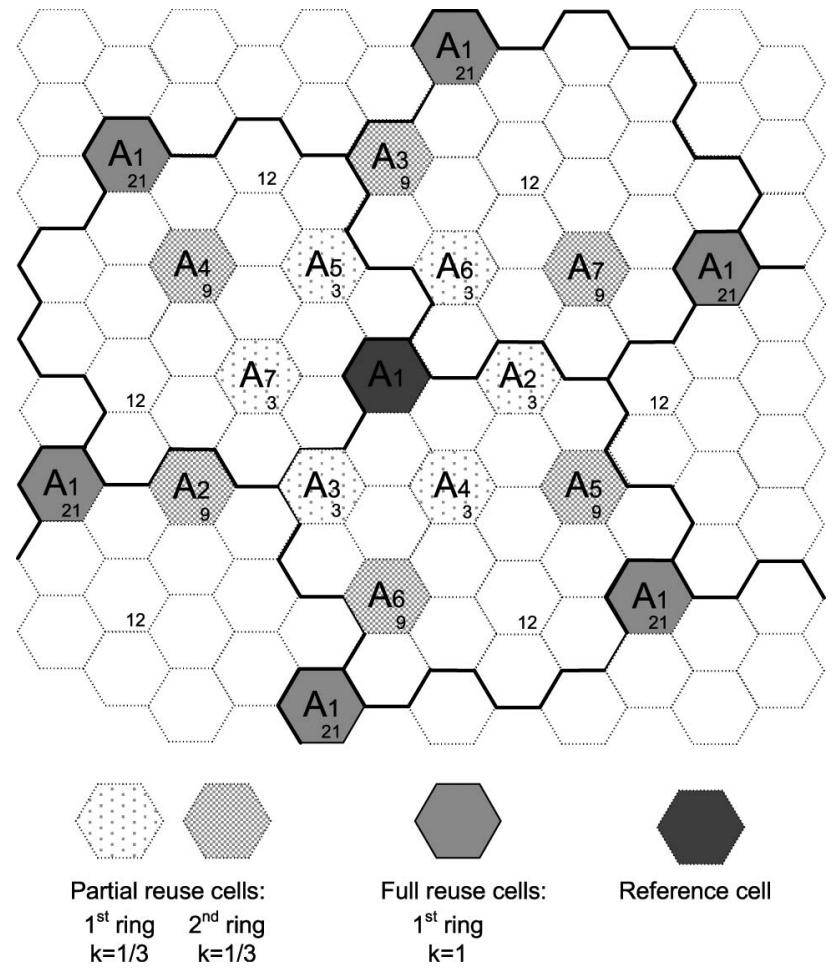

Fig. 2. Frequency reuse cells of a 21/3 fractional-reuse structure.

SFHMA system. Furthermore, the spectral efficiency of the STFSKaided SFHMA, the Gaussian minimum shift keying (GMSK)-aided SFHMA, and the conventional GMSK-aided TD/FDMA systems will be compared. In this paper, we focus our attention on the base-tomobile downlink (DL).

To calculate the ASE of the entire system, we first consider the probability of correct reception of a hop $q_{c}$, ensured by the forward error correction (FEC) coding and by the audio/video codec of a multimedia session to guarantee an acceptable service quality. According to [3], a $q_{c}$ value of 0.7 can guarantee an acceptable speech quality for a system that utilizes the RS $(8,4)$ channel code and a 16-kbit/s subband codec, resulting in an RS-decoded BER of approximately $3 \times 10^{-3}$. Note that the state-of-the-art AMR speech codec [17] provides a good speech quality, even at $B E R=10^{-2}$. However, to facilitate reliable system acquisition and synchronization, the target BER of $10^{-3}$ is chosen in this paper. The probability $q_{c}$ of correct reception during a hop may be formulated as

$$
q_{c}=\boldsymbol{P} r o b\{\lambda \geq \gamma\}
$$

where $\gamma$ represents the energy per bit to noise power spectral density ratio $\left(E_{b} / N_{0}\right)$ that corresponds to the bit-error-ratio (BER) threshold, and $\lambda$ is the carrier-to-interference ratio (CIR) at the receiver considered, where the interference is imposed by all the adjacent cells. Note that the CIR depends on the received power, the shadowing and fast-fading parameters, and the interference imposed by other cells in the network. According to [11], (13) may be expressed as

$$
q_{c}=\prod_{i=1}^{M_{I}}\left(1-\frac{p_{i} \gamma}{\Lambda_{i}+\gamma}\right)
$$

where $M_{I}$ denotes the number of cells that impose interference on the cell considered, whereas $\Lambda_{i}$ is the CIR that corresponds to the interference from the $i$ th cell. Finally, $p_{i}$ presents the frequencycollision probability.

In line with [11], we assume that the locations of MSs are random in the reference cell. Therefore, $q_{c}$ may be calculated for various values of the teletraffic load $X$, which is also known as the average number of users that are actively engaged in calls per megahertz per cell. In the analysis in [11], the ' $90 \%$ worst case value' $q_{90}$, defined as the specific value of $q_{c}$, which is exceeded with a probability of $90 \%$, is utilized. The study in [3] suggested that the specific operating point where we have $q_{90}=0.7$ in the graph of $q_{90}$ versus $X$ should be selected to ensure an acceptable reception quality. However, the analysis in [11] indicated that $q_{90}=0.8$ might be a better choice for the minimum $q$ to determine the ASE. Hence, the value of $q_{90}=0.8$ is considered in our following analysis.

The ASE calculation may then be simplified to

$$
\eta=a_{\text {cell }} / W=X
$$

where $a_{\text {cell }}$ is the traffic load, and $W$ is the total allocated bandwidth. Furthermore, the frequency-collision probability may be expressed as

$$
p_{i}=\frac{y_{i} r_{a} W X}{n_{t} n_{\text {call }}}
$$

where $n_{t}$ is the number of slots per time-division multiplexing (TDM) frame, $n_{\text {call }}$ is the number of calls per timeslot, whereas $r_{a}$ is the voice activity ratio (VAR), which is typically assumed to be 0.5 for the DL [3]. For a full-reuse cell cluster of size $C_{c}$, the frequencycollision rate $y_{i}$ becomes $C_{c} / 3 N_{c}$. By contrast, we have $y_{i}=$ $k_{i} M_{c} / L_{c} N_{c}$ for the $3 M / L$ fractional-reuse structure. With regard to the channel spacing $f_{s}$, the number of hopping frequencies assigned to each of the three basic colors is given by $N=W /\left(3 f_{s}\right)$.

Hence, (16) may be simplified to

$$
p_{i}=\frac{C_{c} X r_{a} f_{s}}{n_{t} n_{\text {call }}}
$$

for full-reuse cells and

$$
p_{i}=\frac{3 k_{i} M X r_{a} f_{s}}{L n_{t} n_{\text {call }}}
$$

for fractional reuse.

In reality, most cellular systems employ fixed beam-forming-based angular sectorization, at least near the cell edge. Thus, in the scenario of $120^{\circ}$ sectorization, only three of the six neighbor cells impose interference on the reference cell. However, only two cells contribute the full interference. Moreover, the authors in [3] demonstrated that the probability of the event when the worst case value $q_{90}$ occurs is comparable to the probability of $\Lambda_{90}$, which is defined as the ' $90 \%$ worst case value' CIR; in other words, $\boldsymbol{P} \operatorname{rob}\left(q_{c}>q_{90}\right)=\boldsymbol{P} \operatorname{rob}(\Lambda>$ $\left.\Lambda_{90}\right)=90 \%$. Hence, $q_{90}$ may be expressed as

$$
q_{90}=\prod_{i=1}^{K_{I}}\left(1-\frac{p_{i} \gamma}{2 \Lambda_{i}+\gamma}\right)^{2}
$$

where $K_{I}$ is the number of rings using a different frequency set around the reference cell.

Fig. 3 shows the BER performance of both GMSK and STFSK(4/1/4/2-4-2), where we employ $M / N / T / Q=4 / 1 / 4 / 2$, 4-PSK and 2-FSK modulation for transmission over the COST-207 rural area channel model [18] when assuming a maximum multipath delay of $20 \mu \mathrm{s}$, which corresponds to an intersymbol interference (ISI) of six symbols at a bit rate of about $300 \mathrm{kbits} / \mathrm{s}$. The half-rate channel codes $\operatorname{RS}(8,4)[11]$ and the recursive systematic convolutional (RSC) code $\operatorname{RSC}(23,33)$ using the octally represented generator polynomials 


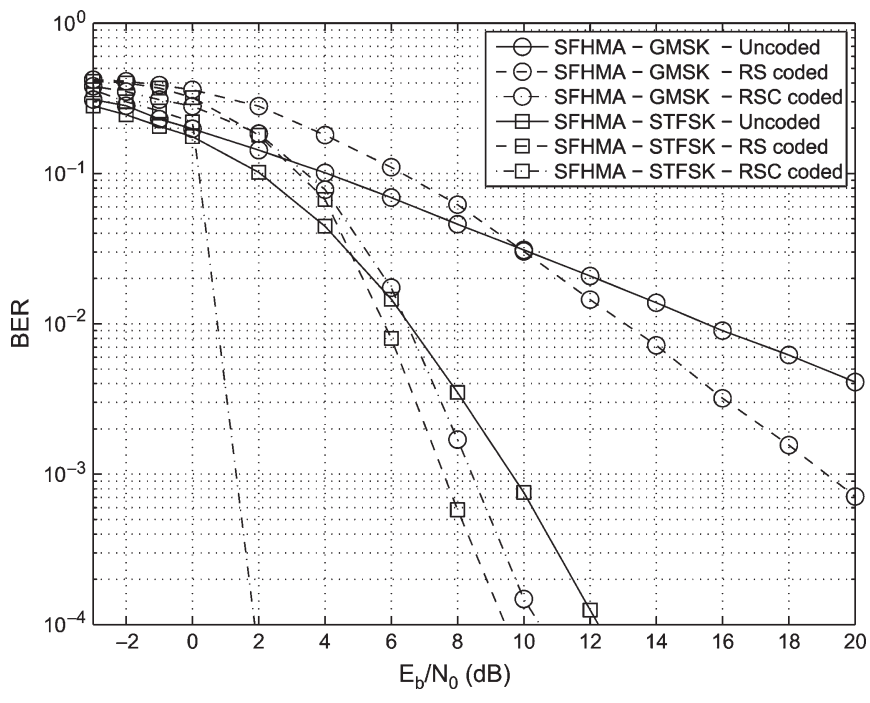

Fig. 3. BER versus $E_{b} / N_{0}$ performance of the GMSK and the STFSK(4/1/4/2-4-2) schemes employing RS and RSC channel codes for transmission over the six-tap COST-207 rural area channel model subjected to Rayleigh fading and additive white Gaussian noise (AWGN).

of 23 and 33 [19] are considered. For the RSC-coded system, soft decision was used at the receiver. According to Fig. 3, the target BER of $10^{-3}$ was achieved at the $E_{b} / N_{0}$ value of $\gamma=26.0,19.0$, and $8.5 \mathrm{~dB}$ for the uncoded, RS-coded, and RSC-coded GMSK schemes, respectively. For the same coding schemes invoked for the STFSK transceiver, the corresponding values are $\gamma=9.5,7.5$, and $1.5 \mathrm{~dB}$, respectively. All of these values will be used for computing the system's $q_{90}$ values and its ASE.

For a basic three-cell structure using the aforementioned values of $\gamma$ and $\Lambda_{90}$ based on [11] and assuming a frequency spacing of $f_{s}=1 / 7 \mathrm{MHz}$, the values of $q_{90}$ versus the average number of users $X$ are presented in Fig. 4. The curves were plotted for the following conditions.

- The nearest reuse ring (cluster size 3) only is marked by the circles.

- The nearest reuse ring plus two nearest rings of cells of other colors that impose adjacent-channel interference, for which the values of $\Lambda_{90}$ based on [3] are $R_{A C}+2 \mathrm{~dB}$ and $R_{A C}+9 \mathrm{~dB}$, where $R_{A C}=17 \mathrm{~dB}$ is the adjacent-channel interference rejection for $f_{s}=1 / 7 \mathrm{MHz}$, is indicated by the down-facing triangles.

- The second reuse ring (size 9) only is indicated by the upward triangles.

- The first five reuse rings (size 3, 9, 12, 21, and 27) is marked by the squares.

As shown in the figure, the tiny gaps between the circle-marked curves and the down-facing-marked curves indicate that the adjacentchannel interference insignificantly affects the cell's performance. By contrast, due to the cochannel interference, the cell's teletraffic load $X$ is reduced by approximately 1 user/cell/MHz at $q_{90}=0.8$ when the number of reuse rings increases from one to five. Based on the $q_{90}$ curves, it is possible to find the system's ASE $\eta_{s y s}$ by spotting the $X$ value, where we have $q_{90}=0.8$. Note that the ASE is quantified in terms of Erlang per cell per megahertz.

Theoretically, the probability of frequency collision $p_{i}$ depends on the following three factors: 1) the fractional overlap between the sets of frequencies in the reuse and reference cell; 2) the VAR; and 3) the mean channel utilization $U$. Thus, $p_{i}$ can be expressed as

$$
p_{i}=k_{i} U r_{a} .
$$

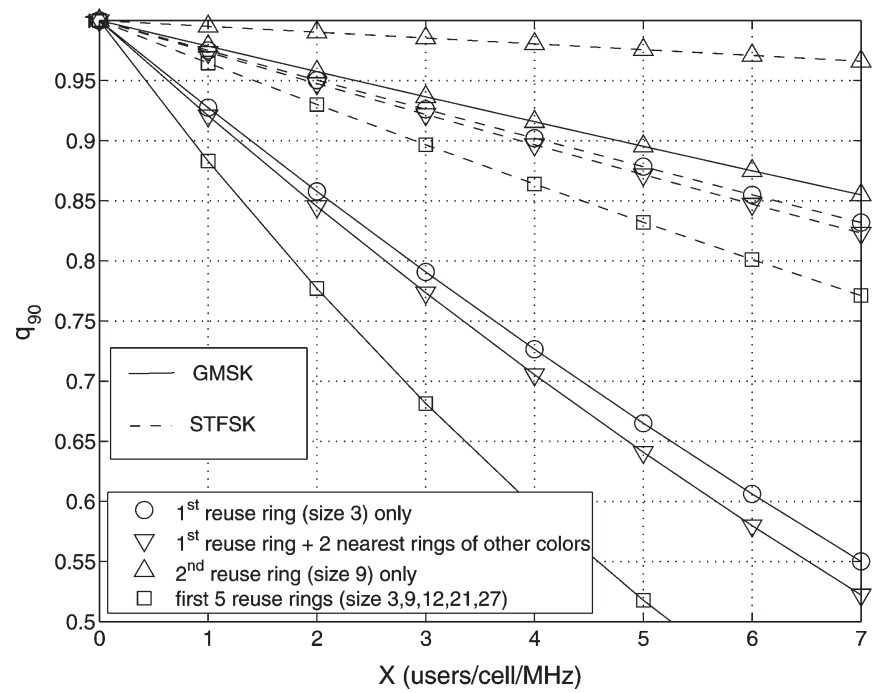

Fig. 4. $\quad q_{90}$ versus $X$ for GMSK and STFSK(4/1/4/2-4-2) employing RSC channel code for a full-reuse cluster size of 3 and $r_{a}=0.5, n_{\text {call }}=1$.

Note that $U=1.0$ when all channels are occupied. Using the condition $U=1.0,(15)$, and (16), the maximum ASE may be obtained as

$$
\eta_{\max }=\frac{n_{t} n_{\text {call }}}{C_{c} f_{s}}
$$

for the full-reuse structure and as

$$
\eta_{\max }=\frac{L_{c} n_{t} n_{\text {call }}}{3 M_{c} f_{s}}
$$

for the fractional-reuse structure.

In practice, the achievable spectral efficiency $\eta_{a c h}$ of a system, which may be achieved with the aid of the Erlang B formula [20], additionally depends on the number of channels per cell $n_{c h}$ and the tolerable blocking probability $P_{B}$. The achievable ASE may be obtained as

$$
\eta_{a c h}=U \times \eta_{\max }
$$

where $U$ may be determined by the Erlang $\mathrm{B}$ equation. Table I shows the maximum and the achievable spectral efficiency of various reuse structures, where we have $W=24 \mathrm{MHz}, f_{s}=1 / 7 \mathrm{MHz}$, and $P_{B}=2 \%$.

Consequently, the attainable ASE of a specific system is given by

$$
\eta_{a t t}=\min \left\{\eta_{\text {sys }}, \eta_{\text {ach }}\right\} \text {. }
$$

The attainable ASE of various reuse structures is provided in Table I. As shown in the table, the STFSK-aided SFHMA may triple the ASE compared to the approach that employs the classic GMSK when no channel coding is employed. When the hard-decision $\operatorname{RS}(8,4)$ channel code was employed, the ASE of the STFSK system is still twice as high as GMSK. Furthermore, through the soft-decision $\operatorname{RSC}(23,33)$, STFSK may approach the maximum achievable ASE for all the reuse structures considered. By contrast, this condition was only possible for the full-reuse cluster size of 9 and for the fractional-reuse cluster size of 21/3 in case of the RSC(23,33)-coded GMSK.

In summary, the attainable ASE can be estimated by the following steps.

- Step 1. Determine the $E_{b} / N_{0}$ threshold $\gamma$ based on Fig. 3, which offers the target BER.

- Step 2. Compute the frequency collision probability $p_{i}$ based on (17) or (18). 
TABLE I

Spectral EfFiciency (IN ERlang Per Cell Per Megahertz) For VARious Reuse Structures: $r_{a}=0.5, P_{B}=2 \%, q 90=0.8$, And $n_{c a l l}=1$

\begin{tabular}{|c|c|c|c|c|c|c|c|c|c|c|}
\hline \multirow{2}{*}{$\begin{array}{c}\text { Cluster } \\
\text { size }\end{array}$} & \multirow[t]{2}{*}{$\eta_{\max }$} & \multirow[t]{2}{*}{$n_{c h}$} & \multirow[t]{2}{*}{$U_{a c h}$} & \multirow[t]{2}{*}{$\eta_{a c h}$} & \multicolumn{2}{|c|}{$\eta_{\text {sys }}$} & \multicolumn{2}{|c|}{$\eta_{a t t}$} & \multicolumn{2}{|c|}{$U \%$} \\
\hline & & & & & Uncoded-GMSK & Uncoded-STFSK & Uncoded-GMSK & Uncoded-STFSK & Uncoded-GMSK & Uncoded-STFSK \\
\hline 3 & 7.00 & 168 & 0.901 & 6.31 & 0.44 & 1.55 & 0.44 & 1.55 & 6 & 22 \\
\hline 9 & 2.33 & 56 & 0.803 & 1.87 & 0.39 & 2.28 & 0.39 & 1.87 & 17 & 80 \\
\hline $9 / 2$ & 4.67 & 112 & 0.872 & 4.07 & 0.43 & 1.70 & 0.43 & 1.70 & 9 & 36 \\
\hline $12 / 2$ & 3.50 & 84 & 0.846 & 2.96 & 0.40 & 1.37 & 0.40 & 1.37 & 11 & 39 \\
\hline $12 / 3$ & 5.25 & 126 & 0.881 & 4.63 & 0.44 & 1.64 & 0.44 & 1.64 & 8 & 31 \\
\hline $21 / 3$ & 3.00 & 71 & 0.831 & 2.49 & 0.41 & 1.77 & 0.41 & 1.77 & 14 & 59 \\
\hline \multirow[t]{2}{*}{$21 / 4$} & 4.00 & 96 & 0.859 & 3.44 & 0.42 & 1.67 & 0.42 & 1.67 & 11 & 42 \\
\hline & & & & & RS-GMSK & RS-STFSK & RS-GMSK & RS-STFSK & RS-GMSK & RS-STFSK \\
\hline 3 & 7.00 & 168 & 0.901 & 6.31 & 0.59 & 2.07 & 0.59 & 2.07 & 8 & 30 \\
\hline 9 & 2.33 & 56 & 0.803 & 1.87 & 0.58 & 3.40 & 0.58 & 2.33 & 25 & 80 \\
\hline $9 / 2$ & 4.67 & 112 & 0.872 & 4.07 & 0.60 & 2.31 & 0.60 & 2.31 & 13 & 49 \\
\hline $12 / 2$ & 3.50 & 84 & 0.846 & 2.96 & 0.51 & 1.85 & 0.51 & 1.85 & 15 & 53 \\
\hline $12 / 3$ & 5.25 & 126 & 0.881 & 4.63 & 0.60 & 2.20 & 0.60 & 2.20 & 11 & 42 \\
\hline $21 / 3$ & 3.00 & 71 & 0.831 & 2.49 & 0.60 & 2.41 & 0.60 & 2.41 & 20 & 80 \\
\hline \multirow[t]{2}{*}{$21 / 4$} & 4.00 & 96 & 0.859 & 3.44 & 0.60 & 2.25 & 0.60 & 2.25 & 15 & 56 \\
\hline & & & & & RSC-GMSK & RSC-STFSK & RSC-GMSK & RSC-STFSK & RSC-GMSK & RSC-STFSK \\
\hline 3 & 7.00 & 168 & 0.901 & 6.31 & 1.78 & 6.04 & 1.78 & 6.04 & 25 & 86 \\
\hline 9 & 2.33 & 56 & 0.803 & 1.87 & 2.77 & $>7.0$ & 1.87 & 1.87 & 80 & 80 \\
\hline $9 / 2$ & 4.67 & 112 & 0.872 & 4.07 & 1.97 & 6.98 & 1.97 & 4.07 & 42 & 87 \\
\hline $12 / 2$ & 3.50 & 84 & 0.846 & 2.96 & 1.59 & 5.53 & 1.59 & 2.96 & 45 & 85 \\
\hline $12 / 3$ & 5.25 & 126 & 0.881 & 4.63 & 1.88 & 6.50 & 1.88 & 4.63 & 36 & 88 \\
\hline $21 / 3$ & 3.00 & 71 & 0.831 & 2.49 & 2.05 & $>7.0$ & 2.05 & 2.49 & 68 & 83 \\
\hline $21 / 4$ & 4.00 & 96 & 0.859 & 3.44 & 1.92 & 6.64 & 1.92 & 3.44 & 48 & 86 \\
\hline
\end{tabular}

- Step 3. Calculate $q_{90}$ from (19).

- Step 4. Determine the system's ASE $\eta_{\text {sys }}$ by looking for the operating point in Fig. 4, where we have the target $q_{90}$, i.e., $q_{90}=0.8$ in the graph of $q_{90}$ versus $X$.

- Step 5. Calculate the achievable spectral efficiency $\eta_{a c h}$ from (23).

- Step 6. Determine the attainable ASE $\eta_{a t t}$, which is the lower of the pair $\left(\eta_{s y s}, \eta_{a c h}\right)$.

\section{B. Comparison Between the SFHMA and TD/FDMA Systems}

Finally, we compare the attainable performance of the STFSKaided SFHMA to the conventional GMSK-aided TD/FDMA, the thirdgeneration WCDMA, and the fourth-generation LTE systems. The system parameters considered are provided in Table II. To support a voice channel that employs the AMR speech codec operated, for example, at $12.2 \mathrm{~kb} / \mathrm{s}$ in the 5-MHz-bandwidth WCDMA system, a maximum of 98 users can be separated when using a spreading factor of 128 [21]. For the same bandwidth of $5 \mathrm{MHz}$, the LTE system can support up to 200 users [22]. Naturally, both the WCDMA and the LTE, as well as the STFSK system, can support a significantly higher data rate than the $12.2-\mathrm{kb} / \mathrm{s}$ speech rate. However, in this paper, we focused our attention on the telephony service. Therefore, a voice data rate of $12.2 \mathrm{~kb} / \mathrm{s}$ is selected for all systems considered. As a benefit of employing code-division multiple access (CDMA) in WCDMA and orthogonal frequency-division multiplexing (OFDM) in LTE, both systems can operate at full frequency reuse in all the adjacent cells.

The performance of the three systems recorded at an $E_{b} / N_{0}$ of $30 \mathrm{~dB}$ is shown in Fig. 5 in terms of the BER versus the mean CIR. Based on Fig. 5, the threshold BER of $10^{-3}$ is achieved at the mean CIR of $\Lambda_{t h}=12,6.5$, and $-1.0 \mathrm{~dB}$ for the GMSK-aided TD/FDMA, for GSMK-assisted SFHMA, and TSFSK-aided SFHMA systems, respectively. These values will be used for determining the minimum reuse cluster size. More particularly, the selected reuse cluster size $\Lambda_{90}$ must be higher than the threshold mean CIR $\Lambda_{t h}$. In other words, the interference power imposed by frequency-reuse cells on the reference cell must be lower than the critical threshold interference level, which may be calculated from the threshold mean CIR $\Lambda_{t h}$. For example, to satisfy the condition of $\Lambda_{90}>\left(\Lambda_{t h}=10.0 \mathrm{~dB}\right)$, the TD/FDMA's smallest reuse cluster size must be the full-reuse nine-cell cluster, where we have $\Lambda_{90}=13.0 \mathrm{~dB}$ based on [11]. Similarly, based on this table, the minimum cluster size is 3 for both the GMSK- and STFSKaided SFHMA systems.

Applying the aforementioned six-step process, the ASEs of various systems are shown in Table II, last line. According to this table, the GMSK-aided TD/FDMA system does not perform as well as the GMSK-aided SFHMA arrangement in terms of the ASE, which is in contrast to the results in [11]. The reason for this fact is that, when strong channel codes such as the RSC or turbo codes are employed, the reference BER value can be reduced, resulting in smaller reuse cluster sizes. The advantage of small reuse cluster sizes in the SFHMA system becomes less dominant. Moreover, due to the employment of SFH, the SFHMA system requires a longer time slot for each user. Hence, the GMSK-aided SFHMA system performs less efficiently than the GMSK-aided TD/FDMA system. However, the cell's performance may significantly be improved by employing STFSK. Quantitatively, the STFSK-aided SFHMA system attained a spectral efficiency of 24.7 Erl/cell/MHz, outperforming an ASE of $15.0 \mathrm{Erl} / \mathrm{cell} / \mathrm{MHz}$, which was recorded for the GMSK-aided TD/FDMA system. This ASE is also significantly higher than the WCDMA's ASE of 16.9 Erl/cell/MHz, which is only slightly better than the GMSK-aided TD/FDMA system. Finally, the LTE system achieves an ASE of 36.5 Erl/cell/MHz, hence exhibiting a substantially better ASE than all the remaining systems considered. In the MIMO configurations, the LTE systems employ space-time block codes (STBCs) to achieve diversity gain or spatial multiplexing schemes to achieve multiplexing gain. As mentioned in Section I, a flexible tradeoff may be struck between the achievable diversity gain and the multiplexing gain by employing linear dispersion code (LDC). As demonstrated in [23], our STFSK schemes can halve the decoding complexity of the LDC schemes upon using the same coding rate. Furthermore, the LTE employs the highly complex OFDM [22]. Therefore, the better performance of 
TABLE II

Spectral Efficiency (in Erlang Per Cell per Megahertz) for Various Systems: $E_{b} / N_{0}=30 \mathrm{~dB}$ and $P_{B}=2 \%$

\begin{tabular}{|c|c|c|c|c|c|c|}
\hline \multicolumn{2}{|l|}{ System parameters } & \multirow{2}{*}{$\begin{array}{r}\text { TD-FDMA } \\
\text { GMSK }\end{array}$} & \multicolumn{2}{|c|}{ SFHMA } & \multirow[t]{2}{*}{ WCDMA } & \multirow[t]{2}{*}{ LTE } \\
\hline & & & GMSK & STFSK & & \\
\hline Speech codec & & $\begin{array}{r}\text { AMR } \\
(12.2 \mathrm{kbps})\end{array}$ & $\begin{array}{r}\text { AMR } \\
(12.2 \mathrm{kbps})\end{array}$ & $\begin{array}{r}\text { AMR } \\
(12.2 \mathrm{kbps})\end{array}$ & $\begin{array}{r}\text { AMR } \\
(12.2 \mathrm{kbps})\end{array}$ & $\begin{array}{r}\text { AMR } \\
(12.2 \mathrm{kbps})\end{array}$ \\
\hline Channel code type & & $\operatorname{RSC}(23,33)$ & $\operatorname{RSC}(23,33)$ & $\operatorname{RSC}(23,33)$ & $\operatorname{RSC}(23,33)$ & $\operatorname{RSC}(23,33)$ \\
\hline Modulation type & & GMSK & GMSK & $\begin{array}{r}\text { STFSK } \\
(4 / 1 / 4 / 2-4-2)\end{array}$ & QPSK & QPSK \\
\hline Antenna configuration ( $\mathrm{Tx}$ ) & & $1 / 1$ & $1 / 1$ & $4 / 1$ & $1 / 1$ & $4 / 1$ \\
\hline System bandwidth (MHz) & $W$ & 5 & 5 & 5 & 5 & 5 \\
\hline Cluster size & $C$ & 9 & 9 & 3 & 1 & 1 \\
\hline No. of timeslots & $n_{t}$ & 8 & 3 & 3 & 1 & 1 \\
\hline No. of call per timeslot & $n_{\text {call }}$ & 4 & 4 & 4 & 1 & 1 \\
\hline Frequency spacing (MHz) & $f_{s}$ & 0.200 & 0.143 & 0.143 & 5 & 5 \\
\hline No. of frequencies/system & $n_{f_{s}}$ & 25 & 35 & 168 & 1 & 1 \\
\hline Spreading factor & & 1 & 1 & 1 & 128 & 512 \\
\hline No. of channels/cell & $n_{c h}$ & 89 & 47 & 140 & 98 & 200 \\
\hline Traffic/channel $(e r l)$ & $a_{c h}$ & 0.841 & 0.768 & 0.883 & 0.860 & 0.912 \\
\hline Traffic/cell (erl) & $a_{\text {cell }}$ & 74.85 & 35.80 & 123.48 & 84.31 & 182.43 \\
\hline $\begin{array}{l}\text { Spectral efficiency } \\
(\mathrm{Erl} / \mathrm{cell} / \mathrm{MHz})\end{array}$ & $\eta_{a t t}$ & 14.958 & 7.161 & 24.697 & 16.86 & 36.487 \\
\hline
\end{tabular}

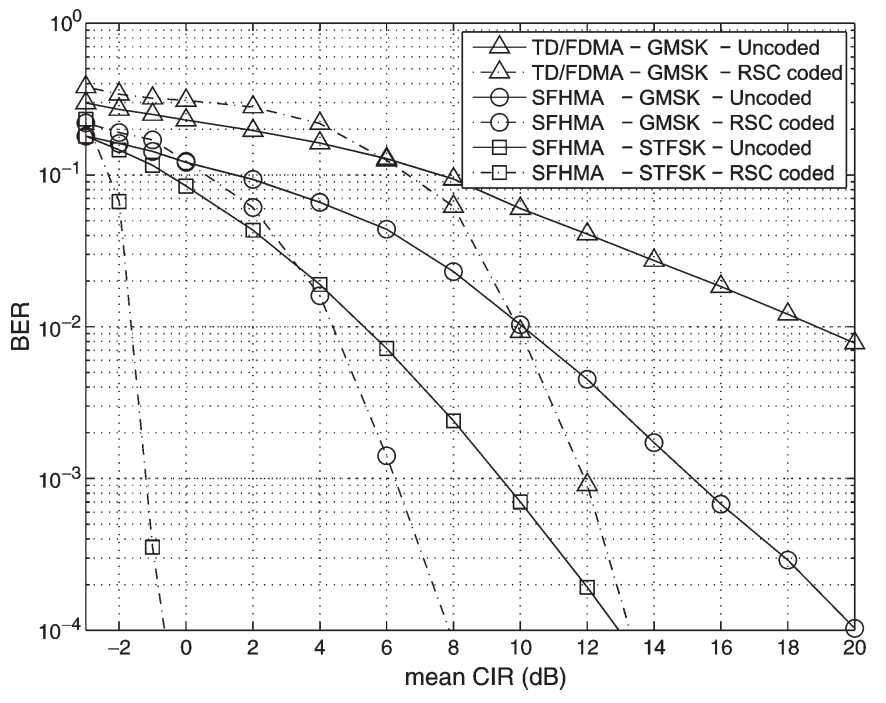

Fig. 5. BER versus mean CIR for the GMSK-aided TD/FDMA, GMSKaided SFHMA, and STFSK(4/1/4/2-4-2)-aided SFHMA systems, where RSC channel codes are employed in a Rayleigh fading AWGN channel at $E_{b} / N_{0}=$ $30 \mathrm{~dB}$.

LTE system is achieved at the cost of a significantly higher system complexity.

\section{CONCLUSION}

In this paper, we have investigated the ASE of the proposed STFSKaided SFHMA system in various frequency reuse structures. The results showed that the proposed system may double the attainable ASE compared to the GMSK-aided SFHMA when RS $(8,4)$ channel codes are employed for transmission over the six-tap COST-207 rural area channel model associated with Rayleigh fading and AWGN. In addition, the soft-decision RSC $(23,33)$-coded STFSK-aided SFHMA may approach the maximum achievable ASE in various frequency reuse cluster sizes. By contrast, this condition is possible only for a full-reuse cluster size of 9 and a fractional-reuse cluster size of 21/3 in case of the soft-decision RSC $(23,33)$-coded STFSK-aided SFHMA. Furthermore, the system's ASE was compared to the soft-decision RSC-coded GMSK-aided TD/FDMA. We demonstrated that the softdecision RSC(23,33)-coded STFSK-aided SFHMA system can outperform the ASE of the RSC(23,33)-coded GMSK-aided TD/FDMA and WCDMA. Despite this significant improvement, the ASE of the STFSK-aided SFHMA remains only $60 \%$ of the more complex LTE system when the same RSC $(23,33)$ channel code and a system bandwidth of $5 \mathrm{MHz}$ are employed. Hence, in our future research, we will find appropriate upper layer techniques for STFSK in the interest of increasing its ASE.

\section{REFERENCES}

[1] E. Geraniotis and M. Pursley, "Error probabilities for slow-frequencyhopped spread-spectrum multiple-access communications over fading channels," IEEE Trans. Commun., vol. COM-30, no. 5, pp. 996-1009, May 1982.

[2] D. Verhulst, "Spectrum efficiency analysis of the digital system SFH900," in Proc. 2nd Nordic Semin. Digit. Land Mobile, Stockholm, Sweden, Oct. 1986, pp. 14-16.

[3] J.-L. Dornstetter and D. Verhulst, "Cellular efficiency with slow frequency hopping: Analysis of the digital SFH900 mobile system," IEEE J. Sel. Areas Commun., vol. SAC-5, no. 5, pp. 835-848, Jun. 1987.

[4] G. J. Foschini, "Layered space-time architecture for wireless communication in a fading environment when using multielement antennas," Bell Lab. Tech. J., vol. 1, no. 2, pp. 41-59, 1996.

[5] P. W. Wolniansky, G. J. Foschini, G. D. Golden, R. A. Valenzuela, "V-BLAST: An architecture for realizing very high data rates over the rich-scattering wireless channel," in Proc. URSI ISSSE, 1998, pp. 295-300.

[6] S. M. Alamouti, "A simple transmit diversity technique for wireless communications," IEEE J. Sel. Areas Commun., vol. 16, no. 8, pp. 1451-1458, Oct. 1998.

[7] V. Tarokh, N. Seshadri, and A. R. Calderbank, "Space-time codes for high-data-rate wireless communication: Performance criterion and code construction," IEEE Trans. Inf. Theory, vol. 44, no. 2, pp. 744-765, Mar. 1998.

[8] R. Y. Mesleh, H. Haas, S. Sinanovic, C. W. Ahn, and S. Yun, "Spatial modulation," IEEE Trans. Veh. Technol., vol. 57, no. 4, pp. 2228-2241, Jul. 2008

[9] J. Jeganathan, A. Ghrayeb, L. Szczecinski, and A. Ceron, "Space shiftkeying modulation for MIMO channels," IEEE Trans. Wireless Commun., vol. 8, no. 7, pp. 3692-3703, Jul. 2009.

[10] H. A. Ngo, C. Xu, S. Sugiura, and L. Hanzo, "Space-time-frequency shift keying for dispersive channels," IEEE Signal Process. Lett., vol. 18, no. 3, pp. 177-180, Mar. 2011.

[11] R. Steele and L. Hanzo, Mobile Radio Communications: Second-and Third-Generation Cellular and WATM Systems, 2nd ed. New York: Wiley-IEEE Press, May 1999.

[12] J. G. Proakis, Digital Communications, 4th ed. New York: McGrawHill, 2001.

[13] J. Jeganathan, A. Ghrayeb, and L. Szczecinski, "Spatial modulation: Optimal detection and performance analysis," IEEE Commun. Lett., vol. 12, no. 8 , pp. 545-547, Aug. 2008. 
[14] T. L. Marzetta and B. M. Hochwald, "Capacity of a mobile multipleantenna communication link in Rayleigh flat fading," IEEE Trans. Inf. Theory, vol. 45, no. 1, pp. 139-157, Jan. 1999.

[15] B. M. Hochwald and W. Sweldens, "Differential unitary space-time modulation," IEEE Trans. Commun., vol. 48, no. 12, pp. 2041-2052, Dec. 2000.

[16] S. Sugiura, S. Chen, and L. Hanzo, "Coherent and differential space-time shift keying: A dispersion matrix approach," IEEE Trans. Commun., vol. 58, no. 11, pp. 3219-3230, Nov. 2010.

[17] L. Hanzo, C. Somerville, and J. Woodard, Voice and Audio Compression for Wireless Communications, 2nd ed. New York: Wiley-IEEE Press, Oct. 2007.

[18] M. Patzold, Mobile Fading Channels: Modelling, Analysis and Simulation. New York: Wiley, 2001.

[19] European Telecommunications Standards Institute, Digital Cellular Telecommunications System (Phase 2+): Channel Coding, 1999.

[20] International Telecommunication Union-ITU, Teletraffic Engineering Handbook, Jun. 2001.

[21] 3GPP Specifications-Series 25: Radio aspects of 3G, including UMTS, 2001.

[22] 3GPP Specifications-Series 36: LTE (Evolved UTRA) and LTEAdvanced Radio Technology-Release 8, 2007.

[23] H. A. Ngo, S. Sugiura, and L. Hanzo, "Soft-demodulation of spacetime-frequency shift keying for iterative detection," in Proc. IEEE ICC, 2012, to be published.

\section{Multiuser Diversity With Adaptive Modulation in Non-Identically Distributed Nakagami Fading Environments}

Anlei Rao, Student Member, IEEE, and

Mohamed-Slim Alouini, Fellow, IEEE

\begin{abstract}
In this paper, we analyze the performance of adaptive modulation with single-cell multiuser scheduling over independent but not identical distributed (i.n.i.d.) Nakagami fading channels. Closed-form expressions are derived for the average channel capacity, spectral efficiency, and bit error rate (BER) for both constant-power variable-rate and variable-power variable-rate uncoded/coded M-ary quadrature amplitude modulation (M-QAM) schemes. We also study the impact of time delay on the average BER of adaptive M-QAM. Selected numerical results show that the multiuser diversity brings considerably better performance, even over i.n.i.d. fading environments.
\end{abstract}

Index Terms-Adaptive modulation, multiuser diversity, Nakagami fading.

\section{INTRODUCTION}

To meet the growing demand of services for wireless communications with relatively scarce spectrum, adaptive modulation [1] has been proposed in the last two decades as an effective method of increasing link spectral efficiency. When the channel side information can be estimated and this estimation can be sent back to the transmitter,

Manuscript received June 7, 2011; revised October 24, 2011; accepted January 18, 2012. Date of publication January 27, 2012; date of current version March 21, 2012. This work was supported by King Abdullah University of Science and Technology. This paper was presented in part at the Eighth International Symposium on Wireless Communication Systems, Aachen, Germany, November 2011. The review of this paper was coordinated by Prof. J. Wu.

The authors are with the Division of Physical Sciences and Engineering, King Abdullah University of Science and Technology, Thuwal 23955-6900, Saudi Arabia (e-mail: fanlei.rao@kaust.edu.sa; slim.alouinig@kaust.edu.sa).

Color versions of one or more of the figures in this paper are available online at http://ieeexplore.ieee.org.

Digital Object Identifier 10.1109/TVT.2012.2185965 the transmit rate and power can be adapted relative to the channel characteristics to achieve better performance. In [2], channel capacity with channel side information was derived for both constant-power variable-rate (CPVR) and variable-power variable-rate (VPVR) transmissions. In [3], a VPVR M-ary quadrature amplitude modulation (M-QAM) scheme was proposed for data transmission over fading channels. This paper showed that there is a constant power gap between the channel capacity and the spectral efficiency for this scheme, and this gap is a function of the maintained bit error rate (BER). In [4], variable-rate adaptation for M-QAM with constant-power allocation has been analyzed over the Nakagami fading channels.

In a single-cell multiuser environment, multiuser diversity [5] takes advantage of the variability in fading channels to improve the overall spectral efficiency. For instance, consider that a reasonably large number of users experiencing independent time-varying fading conditions are actively transmitting or receiving data in a given cell. By granting channel access only to the user with the best transmission quality, we can achieve a diversity gain so that an increase in the channel capacity and spectral efficiency can be reached. Different multiuser scheduling algorithms were proposed in [5] and [6] to choose the best user and reduce the feedback load. In this paper, we simply select the user with the largest instantaneous signal-to-noise ratio (SNR) to perform data transmission. With the best user selected, we analyze the average channel capacity, spectral efficiency, and BER for both CPVR and VPVR schemes with M-QAM over independent but not necessarily identical distributed (i.n.i.d.) Nakagami fading channels.

The operation of the CPVR and VPVR schemes needs accurate channel estimation at the receiver, a reliable feedback path between the estimator and transmitter, and negligible time delay. Thus, an efficient error control scheme is therefore needed to ensure the transmitter's access of channel side information. In this paper, we assume perfect channel estimation and a reliable feedback, and we also quantify the effect of time delay on the proposed system under consideration. In addition, it has been shown in [4] that the time delay affects the average BER due to the variability of the channel conditions. As such, the goal is to keep the time delay under a threshold to limit the impact on the BER.

The rest of this paper is organized as follows: In Section II, the multiuser model is introduced. Next, Section III first reviews the channel capacity and spectral efficiency of both the CPVR and VPVR schemes and then gives closed-form expressions for them with best user selection over i.n.i.d. Nakagami fading channels. The performance of the adaptive M-QAM is also analyzed in this section. In Section IV, we consider the impact of time delay on the average BER. Finally, some numerical results are shown in Section V, and conclusions are drawn in the last section.

\section{System ModeL}

Consider a single-cell multiuser environment with $N$ users uniformly distributed in a cell of radius $R$ centered around a base station. In this case, the average SNR $\bar{\gamma}$ for each user is assumed to be lognormal distributed with a shadow standard deviation of $\theta \mathrm{dB}$ and an average SNR $\bar{\gamma}_{R}$ at distance $R$, as well as a mean value following an exponentially decreasing path-loss model with an exponent $\zeta$. The probability density function (pdf) for the average $\mathrm{SNR} \bar{\gamma}$ is given by [7], [8]

$$
f_{\bar{\gamma}}(\bar{\gamma})=\frac{2}{c} \exp \left\{\frac{2 \theta^{2}-2 c\left(\bar{\gamma}-\bar{\gamma}_{R}\right)}{c^{2}}\right\} Q\left(\frac{2 \theta^{2}-c\left(\bar{\gamma}-\bar{\gamma}_{R}\right)}{c \cdot \theta}\right)
$$

